\title{
Comparison of two transurethral endoscopic modalities used in treatment of bladder stones: a systematic review and meta-analysis for randomized controlled trials
}

\author{
Liping Gou \\ West China Hospital of Sichuan University \\ Zhenghao Wang \\ West China Hospital of Sichuan University \\ Ye Zhou \\ West China Hospital of Sichuan University \\ Xiaofeng Zheng ( $\square$ xiaofeng.zheng@wchscu.cn ) \\ West China Hospital of Sichuan University
}

\section{Research Article}

Keywords: bladder stones, transurethral, endoscopic treatments, cystoscope, nephroscope

Posted Date: July 24th, 2021

DOI: https://doi.org/10.21203/rs.3.rs-651932/v1

License: (c) (1) This work is licensed under a Creative Commons Attribution 4.0 International License.

Read Full License 


\section{Comparison of two transurethral endoscopic modalities used in treatment of bladder stones: a systematic review and meta-analysis for randomized controlled trials}

Liping Gou ${ }^{1}$, Zhenghao Wang ${ }^{1}$, Ye Zhou ${ }^{1}$, Xiaofeng Zheng ${ }^{1 *}$

1. Center for diabetes and metabolism research, Division of Endocrinology and metabolism, West China Hospital, Sichuan University, Chengdu, P R China

*Correspondence: Xiaofeng Zheng, Center for diabetes and metabolism research, Division of Endocrinology and metabolism, West China Hospital, Sichuan University, Chengdu, Xinchuan road 222\# 610041, China. Email: xiaofeng.zheng@wchscu.cn, Tel: +86 1982029526 Fax: 2885422451. 


\section{Abstract :}

Background: A systematic review and meta-analysis was conducted to compare the safety and efficiency of nephroscope and cystoscope in transurethral cystolithotripsy (TUCL) for bladder stones.

Methods: PubMed, Web of Science, Embase, EBSCO, and the Cochrane library databases (updated January 2021) were searched for studies that assessed the efficacy of different types of endoscopes in TUCL cases. The search strategy and study selection process was managed according to the PRISMA statement.

Results: Five randomized controlled trials were included in the meta-analysis. Our result showed no significant differences in the stone free rate (SFR) across the studies $(R R=1.00$; CI $0.98-1.02, \mathrm{p}=1.00)$. The study heterogeneity ( $2=0 \%, \mathrm{P}=1.00)$ was not significant as all patients were rendered stone free. Using nephroscope can significantly shorten operation times compared to the use of cystoscopy $(R R=-26.26$; CI $-35.84--16.68, p<0.00001)$ with significant heterogeneity (I2 $=87 \%, \mathrm{p}<0.00001)$. There was no significant difference between mean urethral entries $(\mathrm{RR}=0.66$; $\mathrm{CI}-0.71--2.04, \mathrm{p}=0.35)$, hospitalization $(\mathrm{MD}=$ $0.08,95 \% \mathrm{CI}=-0.07-0.23 ; \mathrm{P}=0.31)$ and the total complication rate $(\mathrm{RR}=1.37,95 \% \mathrm{CI}=$ $0.47-4.00, \mathrm{p}=0.56)$.

Conclusions: This systematic review demonstrated that using are highly efficient approaches in the clearance of bladder stones and result in a low rate of complication and short hospitalization times. The mean urethral entries are dependent on the treatment method for large stone fragments. However, using nephroscope can significantly reduce operation times.

Key words: bladder stones; transurethral; endoscopic treatments; cystoscope; nephroscope 


\section{Background}

Recent improvements in modern antibiotic treatments and improved nutrition have led to a decrease in the incidence of bladders stones (BS) [1]. BS remain the most common type of stone found in the lower urinary tract and account for around 5\% of all urinary calculi [2]. BS are commonly classified as primary, secondary, and migratory types [3] and are often associated with neurogenic voiding dysfunction, bladder outlet obstruction and urinary tract infection [4].

Compared to conservative therapy and chemolysis, surgical approaches are more efficient strategies for the treatment of BS. Several surgical procedures can be used in the treatment of BS including open or transurethral surgery, percutaneous procedures, and extracorporeal shock wave lithotripsy (ESWL)[5]. The development of endoscopic surgical techniques over the past 50 years has resulted in traditional open cystolithotomy (OC) being replaced by minimally invasive treatments [6]. Shock Wave Lithotripsy (SWL) is a simple, well-tolerated and effective approach for the treatment of BS. However, the passage of residual fragments is prolonged and the removal of large stones can be challenging with this intervention [7]. Transurethral cystolithotripsy (TUCL) and percutaneous cystolithotripsy (PCCL) are also frequently used approaches used to treat BS. Several lithotripsy energies including holmium lasers, ultrasound and pneumatic lithotripters are commonly applied for the fragmentation of BS [8]. Despite several different treatment options, the successful treatment of BS remains challenging as the complication rates and operation time varies according to each treatment modality. Also, transurethral surgery is performed using a nephroscope or cystoscope [9] and the choice of endoscope used during surgery may also influence perioperative outcomes.

Previously, a systematic review by Donaldson et al. demonstrated that transurethral endoscopic surgery and percutaneous endoscopic treatments are associated with the same SFR and had shorter operation times, reduced hospital stays and catheterization times compared to OC in patients of all ages. The authors also reported that mechanical, pneumatic, and laser lithotripsy have the same efficiency for transurethral approaches [10]. However, they only reported SFR and operation times in their study for nephroscopy and cystoscopy approaches. Also, one of the included studies used the percutaneous methods to compare the transurethral approach and the total number of studies in the review was limited [11]. A 
comprehensive systematic review focusing on the safety and efficiency of and cystoscope in TUCL for BS is yet to be reported and was the aim of this study.

\section{Materials and methods}

This systematic review and meta-analysis followed the guidelines of the Preferred Reporting Items for Systematic Reviews and Meta-analysis (PRISMA) statement and the Cochrane Handbook for Systematic Reviews of Interventions [12]. Ethical approval and patient consent were not required s all analyses were based on previously published studies.

\section{Literature search and selection criteria}

We systematically searched several databases including PubMed, EMbase, Web of Science, EBSCO, and the Cochrane Library from inception to January 2021 with the following keywords: "bladder," "calculus," "cystoscope," “nephroscope," and "cystoscope”. The reference lists of the retrieved studies and relevant reviews were hand-searched, and the process repeated to ensure that all eligible studies were included in the analysis. Studies reported in all languages were also included.

\section{Data extraction and outcome measures}

Baseline information that was extracted from the original studies included the following: first author, published year, number of patients, patient age and gender distributions, stone burden and detail methods for the two groups, and the evaluation of the evidence level. Data were independently extracted by two investigators. Discrepancies were resolved by consensus.

The primary outcomes were stone free rate (SFR) and operative time. Secondary outcomes are the mean urethral entries, hospitalization, and total complications. 


\section{Quality assessment of individual studies}

All assessments were performed independently by two researchers with differences resolved by discussion. The methodological quality of each RCT was assessed according to the Jadad Scale, which comprises the following three evaluation elements: randomization (0-2 points), blinding (0-2 points), and dropouts and withdrawals (0-1 points)[13]. One point was awarded for each element that was conducted and appropriately described in the original article. The total score varies from 0 to 5 points. An article with a Jadad score of $\leq 2$ is considered to be of low quality, while a Jadad score of $\geq 3$ indicates the high quality of a study[14].

\section{Statistical analysis}

Risk ratios (RR) with 95\% confidence intervals (CIs) were calculated for dichotomous outcomes. Heterogeneity was evaluated using the I 2 statistic, with I $2>50 \%$ taken to indicate significant heterogeneity [15]. Sensitivity analysis was performed for evaluating the influence of a single study on the overall estimate by omitting one study in turn or performing subgroup analysis. The random-effects model was used for meta-analysis. Owing to the limited number of included studies $(<10)$, publication bias was not assessed. Statistical significance was accepted at $\mathrm{P}<0.05$. All statistical analyses were performed using Review Manager Software Version 5.3 (The Cochrane Collaboration, Software Update, Oxford, UK).

\section{Result}

\section{Literature search, study characteristics, and quality assessment}

In total, 102 articles were initially identified from the databases. After removing duplicates, 81 articles were retained. 72 studies were excluded from our study due to unrelated abstracts and titles. We also excluded 5 studies from our analysis as 1 study was not a RCT design, 2 
studies presented insufficient data and 2 studies reported inconformity outcomes. Five RCTs satisfied the inclusion criteria and were enrolled in this meta-analysis [16-20]. The article selection process followed the PRISMA statement (Fig.1). The baseline characteristics of the 5 included studies are shown in Table 1. Four 4 studies used a 24Fr nephroscope and one study used a 22 Fr nephroscope. 4 groups[16-18, 20] used a 22 Fr nephroscope and 1 group used 23 Fr [19] in cystoscopy. All studies used pneumatic lithotripsy with or without ultrasonic lithotripsy as the fragmentation energy. There were no statistically significant differences in the patient baseline characteristics.

All the studies in our meta-analysis were published between 2005 and 2020 and the total sample size was 791. The mean Jadad score ranged from 2 to 5. The main limitations of our methods included the lack of study blinding and description of the specific randomization methods $[16,19,20]$. The Jadad scores for each study are presented in Table 1.

\section{Primary outcomes}

\section{Stone clearance}

All studies reported SFR. Three studies assessed the SFR by KUB or ultrasonography [18-20] and two studies $[16,17]$ did not state the SFR. The results showed no difference in SFR (RR= 1.00; CI 0.98-1.02, $\mathrm{p}=1.00$; Fig. 2) with insignificant heterogeneity ( $\mathrm{I} 2=0 \%, \mathrm{P}=1.00)$ as all patients were rendered stone free.

\section{Operation time}

All studies reported the operation times. The meta-analysis revealed that the nephroscope group had significantly shorter operation times compared to the cystoscopy group $(R R=-$ 26.26; CI -35.84 - -16.68, p < 0.00001; Fig. 3) with significant heterogeneity (I2 =87\%, p < $0.00001)$.

\section{Secondary outcomes}

\section{Mean urethral entries}


Four studies reported the mean urethral entries $[16-18,20]$. Our meta-analysis indicated that there was no difference in the mean urethral entries between the cystoscope and nephroscope groups $(\mathrm{RR}=0.66$; CI $-0.71--2.04, \mathrm{p}=0.35$; Fig. 4) with significant heterogeneity (I2 $=99 \%$, $\mathrm{p}<0.00001)$. Ozdemir et al. only reported the mean number of transurethral access and showed that nephroscope $(18.12 \pm 4.94)$ can significantly reduce the times for transurethral access $(26.81 \pm 3.14)$ compared to cystoscope $(\mathrm{P}<0.0001)[19]$.

\section{Hospitalization}

Three studies [16-18] contained the data of hospitalization and the result indicated that there was no significant difference between the two groups $(\mathrm{MD}=0.08,95 \% \mathrm{CI}=-0.07-0.23$; $\mathrm{P}=0.31)$ with insignificant heterogeneity ( $\mathrm{I} 2=0 \%, \mathrm{P}=0.63$; Fig. 5).

Total complications

Four studies $[16,17,19,20]$ reported data relating to complications. No major complications were reported in the studies. Bansal et al. reported fever, transient hematuria, urethral stricture and Ozdemir et al. [19] reported abrasion of the urethral mucosa with transient hematuria. No significant differences were reported between the two groups $(\mathrm{RR}=1.37,95 \%$ $\mathrm{CI}=0.47-4.00, \mathrm{p}=0.56)$ with insignificant heterogeneity $(\mathrm{I} 2=9 \%, \mathrm{P}=0.29 ;$ Fig. 5).

Sensitivity analysis

Among all outcomes, the operation times and mean urethral entries showed significant heterogeneity $\mathrm{I} 2=87 \%, \mathrm{p}<0.00001 ; \mathrm{I} 2=87 \%, \mathrm{p}<0.00001$ respectively). Sensitivity analysis was performed to evaluate the stability of the results. After removing 1 study at a time, the heterogeneity was $\mathrm{I} 2=89 \%, 91 \%, 85 \%, 88 \%$ and $81 \%$ for the operation times and $\mathrm{I} 2=99 \%$, $99 \%, 99 \%$ and $97 \%$ for the mean urethral entries which indicated that the heterogeneities were stable.

\section{Discussion}

There are currently several different options available for the treatment of BS, however, the selection of the optimum treatment may vary according to individual patient factors [21]. 
Many factors should be taken into consideration when selecting the best treatment option for BS including composition and size of the stone, the general condition of the patient, previous treatment history and specific anatomic abnormalities. Also, the type of surgical equipment is an important factor in therapeutic success [22].

Transurethral surgery has become commonly used due to its high efficacy and low morbidity following the development of newer endoscopic and fragmentation equipment [23]. Many lithotripsy energy facilities can be used for stone crushing such as electrohydraulic lithotripsy, ultrasonic lithotripsy, pneumatic lithotripsy and the holmium:yttrium aluminum-garnet laser can be used via the transurethral approach. The relative advantages and disadvantages of these techniques have been reported in previous studies [24-27]. The diverse types of endoscopes such as cystoscopes and nephroscopes are also widely used in transurethral surgeries. However, the best instrument for BS surgery remains unclear and controversial. In this study, we conducted a systematic review to compare the efficiency and outcomes of these two types of endoscopes in procedures to removing BS.

The complete removal of stone fragments is the most important indicator for evaluating the efficacy of any stone surgery. The main result from our study indicated that there was no difference in SFR between the two types of endoscopes. All of the included patients achieved stone-free status by one session in all type of endoscope (cystoscope and nephroscope) and fragmentation equipment (ultrasonic, pneumatic or both) used during surgery. Donaldson et al. reported that the SFR of TUCL was the same with OC, PCCL and higher than ESWL [10]. Thus, we assumed that the main factor influencing the success rate was the TUCL method and not the devices used during surgery.

Another important evaluation criterion for stone treatment is operation time as removing the fragmented stones from the bladder is time-consuming and the main determining factor for complications and postoperative recovery in TUCL procedure. Our results showed that the mean operation time for stone removal was significantly shorter in the nephroscopy group. A slow inflow of saline can avoid over distension of the bladder during the surgery. In cases where the bladder can become distended, the bladder was emptied by removing the rubber covering over the port inlet of the nephroscope which could provide a better view for the surgeon. Improved views during surgery can facilitate the evacuation of the stone fragments and shorten the operation time [16]. Ener et al. assumed that the nephroscopes had a larger 
lumen compared to cystoscopes and so the nephroscope is better for the removal of the calculus fragments through the lumen which can also shorten the operative time [20].

There was no difference in terms of mean urethral entries. The heterogeneity of this outcome may be due to the different methods of clearing large fragments. Bansal et al. used a cystoscope for the removal of fragments after removing the nephroscope as they used an evacuator for removing large fragments that are only suitable for cystoscopy. After this procedure, the nephroscope was inserted for further fragmentation and retrieval of the fragments [17]. For this reason, the mean urethral entries were much higher in the nephroscope group. Ener et al. and Ozdemir et al. kept the sheath in the urethra to prevent the need for multiple entries to the bladder during surgery during nephroscopy which is an effective and reliable method. However, the removal of larger stone fragments necessitates pulling the cystoscope out with the stone together [19, 20]. Jang et al. reported that larger diameter nephroscopes are easier for the removal of fragmented stones and can significantly reduce the reinsertion of the scope [16].

Our result showed no significant differences in hospitalization and total complication rates indicating that both scopes are safe. No severe complications were reported in all patients highlighting the minimum injury rate of the TUCL. The open and percutaneous approach offered a better view during surgery and prolonged instrumentation of the urethra was avoided. Longer postoperative recovery was associated with a higher incidence of complications due to trauma and placement of the suprapubic catheter [17]. It is also worth noting that the urethral structure is higher $(5 / 70$ vs $2 / 70)$ in the nephroscope group in the study of Bansal et al. though it was not statistically significant. This data might relate to urethral mucosal injury by higher entries of nephroscopy [17].

This study had several limitations. Firstly, the number of included studies and patients is low which may limit the widespread applicability of our data. Secondly, our conclusions may be skewed because of some unpublished data and missing negative results in the original studies. Also, there were differences between the fragmentation equipment and the diameters of the scopes which made assessing the efficacy of surgery difficult. Finally, a large scale RCT with a large sample size and consistent baseline data should be performed in the future to validate our findings. 
In conclusion, this systematic review demonstrates that both nephoscope and cystoscope have high efficiencies for stone clearance, low complication rates and shorter hospitalization stays. The mean urethral entries depend on the treatment method for large stone fragments. However, the use of a nephroscope can significantly reduce operation times.

\section{List of abbreviations}

TUCL transurethral cystolithotripsy

SFR stone free rate

BS bladders stones

ESWL extracorporeal shock wave lithotripsy

OC open cystolithotomy

SWL Shock Wave Lithotripsy

TUCL Transurethral cystolithotripsy

PCCL percutaneous cystolithotripsy

PRISMA Preferred Reporting Items for Systematic Reviews and Meta-analysis

RR Risk ratios

CIs confidence intervals

\section{Acknowledgements}

Not applicable

\section{Competing interests}

The authors declare that there is no competing interests

\section{Funding}

This work is supported by the National Natural Science Foundation of China (82070846) and Program for Overseas High-Level Talents Introduction of Sichuan Province of China (21RCYJ0046). 


\section{Contribution}

GLP, WZH, and ZXF participated in the design of this study. GLP drafted the manuscript. WZH, ZY collected and analysis the data, GLP and ZXF critically revised the manuscript. All authors have read and approved the final manuscript.

\section{Consent for publication}

Not applicable.

\section{Availability of data and materials}

The datasets used and/or analyzed during the current study are available from the corresponding author on reasonable request.

\section{Ethics approval and consent to participate}

Not applicable 


\section{Reference}

1. Wagner CA. Etiopathogenic factors of urolithiasis. Archivos espanoles de urologia. 2021;74(1):16-23. Epub 2021/01/19. PubMed PMID: 33459618.

2. Schwartz BF, Stoller ML. The vesical calculus. Urol Clin North Am. 2000;27(2):333-46. Epub 2000/04/25. doi: 10.1016/s0094-0143(05)70262-7. PubMed PMID: 10778475.

3. Philippou P, Moraitis K, Masood J, Junaid I, Buchholz N. The management of bladder lithiasis in the modern era of endourology. Urology. 2012;79(5):980-6. Epub 2011/11/29. doi: 10.1016/j.urology.2011.09.014. PubMed PMID: 22119259.

4. Yoshida O, Okada Y. Epidemiology of urolithiasis in Japan: a chronological and geographical study. Urol Int. 1990;45(2):104-11. Epub 1990/01/01. doi: 10.1159/000281680. PubMed PMID: 2330656.

5. Cicione A, C DEN, Manno S, Damiano R, Posti A, Lima E, et al. Bladder stone management: an update. Minerva urologica e nefrologica $=$ The Italian journal of urology and nephrology. 2018;70(1):53-65. Epub 2017/10/13. doi: 10.23736/s0393-2249.17.02972-1. PubMed PMID: 29022330.

6. Torricelli FC, Mazzucchi E, Danilovic A, Coelho RF, Srougi M. Surgical management of bladder stones: literature review. Revista do Colegio Brasileiro de Cirurgioes. 2013;40(3):227-33. Epub 2013/08/06. doi: 10.1590/s0100-69912013000300011. PubMed PMID: 23912371.

7. Papatsoris AG, Varkarakis I, Dellis A, Deliveliotis C. Bladder lithiasis: from open surgery to lithotripsy. Urol Res. 2006;34(3):163-7. Epub 2006/02/14. doi: 10.1007/s00240-006-0045-5. PubMed PMID: 16470391.

8. Razvi HA, Song TY, Denstedt JD. Management of vesical calculi: comparison of lithotripsy devices. J Endourol. 1996;10(6):559-63. Epub 1996/12/01. doi: 10.1089/end.1996.10.559. PubMed PMID: 8972793.

9. Toktas G, Sacak V, Erkan E, Kocaaslan R, Demiray M, Unluer E, et al. Novel technique of cytolithotripsy for large bladder stones. Asian journal of endoscopic surgery. 2013;6(3):245-8. Epub 2013/07/25. doi: 10.1111/ases.12029. PubMed PMID: 23879422.

10. Donaldson JF, Ruhayel Y, Skolarikos A, MacLennan S, Yuan Y, Shepherd R, et al. Treatment of Bladder Stones in Adults and Children: A Systematic Review and Meta-analysis on Behalf of the European Association of Urology Urolithiasis Guideline Panel. Eur Urol. 2019;76(3):352-67. Epub 2019/07/18. doi: 10.1016/j.eururo.2019.06.018. PubMed PMID: 31311676.

11. Zhao J, Shi L, Gao Z, Liu Q, Wang K, Zhang P. Minimally invasive surgery for patients with bulky bladder stones and large benign prostatic hyperplasia simultaneously: a novel design. Urol Int. 2013:91(1):31-7. Epub 2013/06/12. doi: 10.1159/000350235. PubMed PMID: 23751405.

12. Liberati A, Altman DG, Tetzlaff J, Mulrow C, Gøtzsche PC, loannidis JP, et al. The PRISMA statement for reporting systematic reviews and meta-analyses of studies that evaluate health care interventions: explanation and elaboration. PLoS medicine. 2009;6(7):e1000100. Epub 2009/07/22. doi: 10.1371/journal.pmed.1000100. PubMed PMID: 19621070; PubMed Central PMCID: PMCPMC2707010 Oxford Radcliffe Hospitals Trust on behalf of the Department of Health and the National Institute for Health Research in England.

13. JADAD A. Assessing the quality of reports of randomized clinical trials : is blinding necessary? Control Clin Trials. 1996;17.

14. Kjaergard LL, Villumsen J, Gluud C. Reported Methodologic Quality and Discrepancies between Large and Small Randomized Trials in Meta-Analyse. Annals of Internal Medicine. 2001;135(11):982-9.

15. Higgins JP, Thompson SG. Quantifying heterogeneity in a meta-analysis. Stat Med. 2002;21(11):153958. Epub 2002/07/12. doi: 10.1002/sim.1186. PubMed PMID: 12111919.

16. Jang JY, Ko YH, Song PH, Choi JY. Comparison of three different endoscopic approaches in the treatment of bladder calculi. Yeungnam University journal of medicine. 2019;36(1):16-9. Epub 2019/10/18. doi: 10.12701/yujm.2019.00045. PubMed PMID: 31620607; PubMed Central PMCID: PMCPMC6784616.

17. Bansal A, Kumar M, Sankhwar S, Goel S, Patodia M, Aeron R, et al. Prospective randomized comparison of three endoscopic modalities used in treatment of bladder stones. Urologia. 2016;83(2):87- 
92. Epub 2016/04/23. doi: 10.5301/uro.5000171. PubMed PMID: 27103095.

18. Singh KJ, Kaur J. Comparison of three different endoscopic techniques in management of bladder calculi. Indian journal of urology : IJU : journal of the Urological Society of India. 2011;27(1):10-3. Epub 2011/07/01. doi: 10.4103/0970-1591.78402. PubMed PMID: 21716932; PubMed Central PMCID: PMCPMC3114570.

19. TunçOzdemir A, Koyuncu H, Altinova S, Asil E, Isgoren E, Gurdal M. Comparison of Transurethral use of Nephroscope with Cystoscope in Transurethral Cystolithotripsy. Journal of Clinical and Analytical Medicine. 2014;5:133-7. doi: 10.4328/JCAM.1248.

20. Ener K, Agras K, Aldemir M, Okulu E, Kayigil O. The randomized comparison of two different endoscopic techniques in the management of large bladder stones: transurethral use of nephroscope or cystoscope? J Endourol. 2009;23(7):1151-5. Epub 2009/06/18. doi: 10.1089/end.2008.0647. PubMed PMID: 19530944.

21. Javali T, Nayak KA, Babu S. Simultaneous antegrade and retrograde endoscopic surgery for benign prostatic hyperplasia with vesical calculi - A single-centre experience. Arab J Urol. 2018;16(4):417-21. Epub 2018/12/12. doi: 10.1016/j.aju.2018.04.004. PubMed PMID: 30534441; PubMed Central PMCID: PMCPMC6277507.

22. Sarkis J, Alkassis M, Chebel JA, Tabcheh A, Semaan A. Bladder stone following intravesical migration of surgical clip five years after radical prostatectomy. Urology case reports. 2020;28:101060. Epub 2019/11/26. doi: 10.1016/j.eucr.2019.101060. PubMed PMID: 31763168; PubMed Central PMCID: PMCPMC6861555.

23. Roslan M, Przudzik M, Borowik M. Endoscopic intact removal of medium-size- or multiple bladder stones with the use of transvesical laparoendoscopic single-site surgery. World J Urol. 2019;37(2):373-8. Epub 2018/06/30. doi: 10.1007/s00345-018-2358-8. PubMed PMID: 29955919; PubMed Central PMCID: PMCPMC6373356.

24. Bülow H, Frohmüller HG. Electrohydraulic lithotripsy with aspiration of the fragments under vision-304 consecutive cases. J Urol. 1981;126(4):454-6. Epub 1981/10/01. doi: 10.1016/s0022-5347(17)545735. PubMed PMID: 7288931.

25. Maeda O, Usami M. [Summary of 'General Rule for Clinical and Pathological Studies on Prostate Cancer (the 3rd Edition)']. Nihon rinsho Japanese journal of clinical medicine. 2005;63(2):201-6. Epub 2005/02/18. PubMed PMID: 15714966.

26. Bhatia V, Biyani CS. A comparative study of cystolithotripsy and extracorporeal shock wave therapy for bladder stones. International urology and nephrology. 1994;26(1):26-31. Epub 1994/01/01. doi: 10.1007/bf02768241. PubMed PMID: 8026920.

27. Matsuoka K, lida S, Nakanami M, Koga H, Shimada A, Mihara T, et al. Holmium: yttrium-aluminumgarnet laser for endoscopic lithotripsy. Urology. 1995;45(6):947-52. Epub 1995/06/01. doi: 10.1016/s0090-4295(99)80113-7. PubMed PMID: 7771028. 


\section{Legend of Figures}

Figure 1. Flow diagram of study searching and selection process.

Figure 2. Forest plot for the meta-analysis of stone clearance.

Figure 3. Forest plot for the meta-analysis of operation time.

Figure 4. Forest plot for the meta-analysis of mean urethral entries.

Figure 5. Forest plot for the meta-analysis of hospitalization.

Figure 6. Forest plot for the meta-analysis of total complication. 


\section{Table 1. Characteristics of included studies}

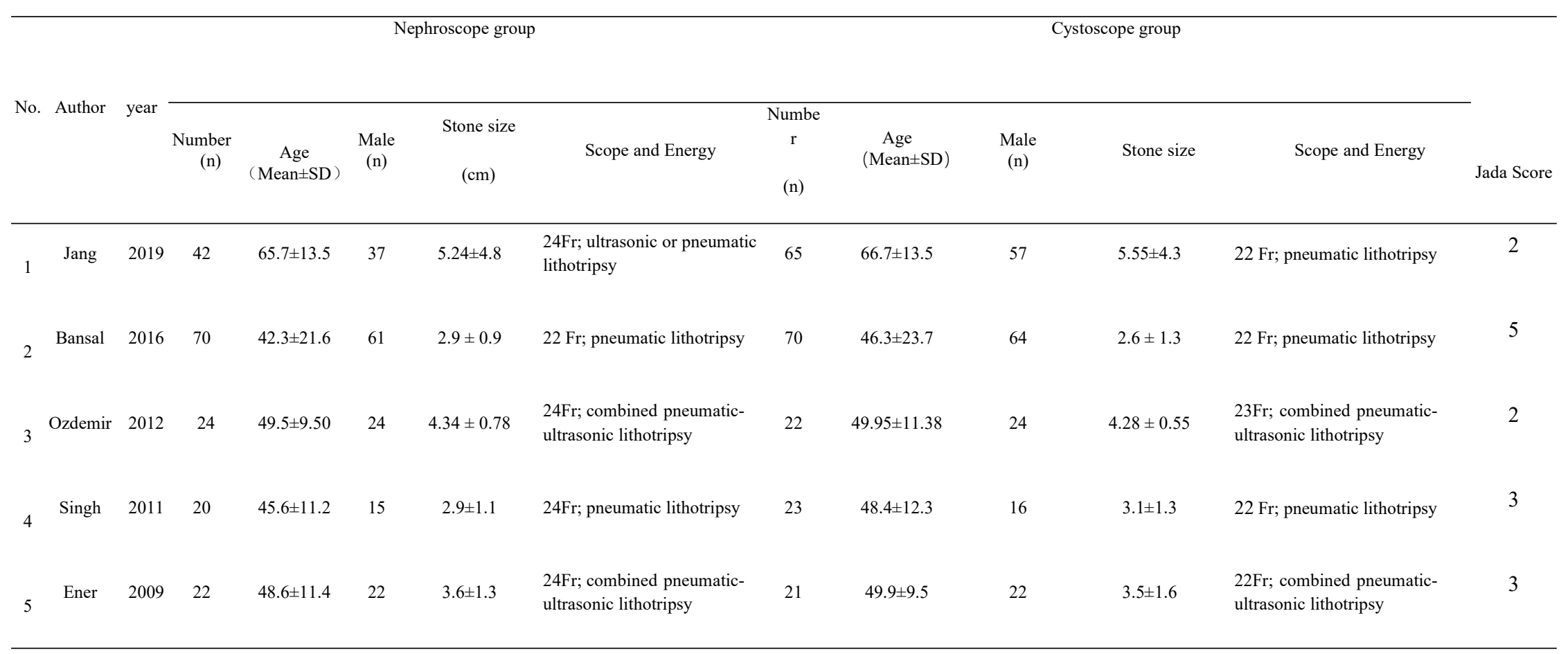




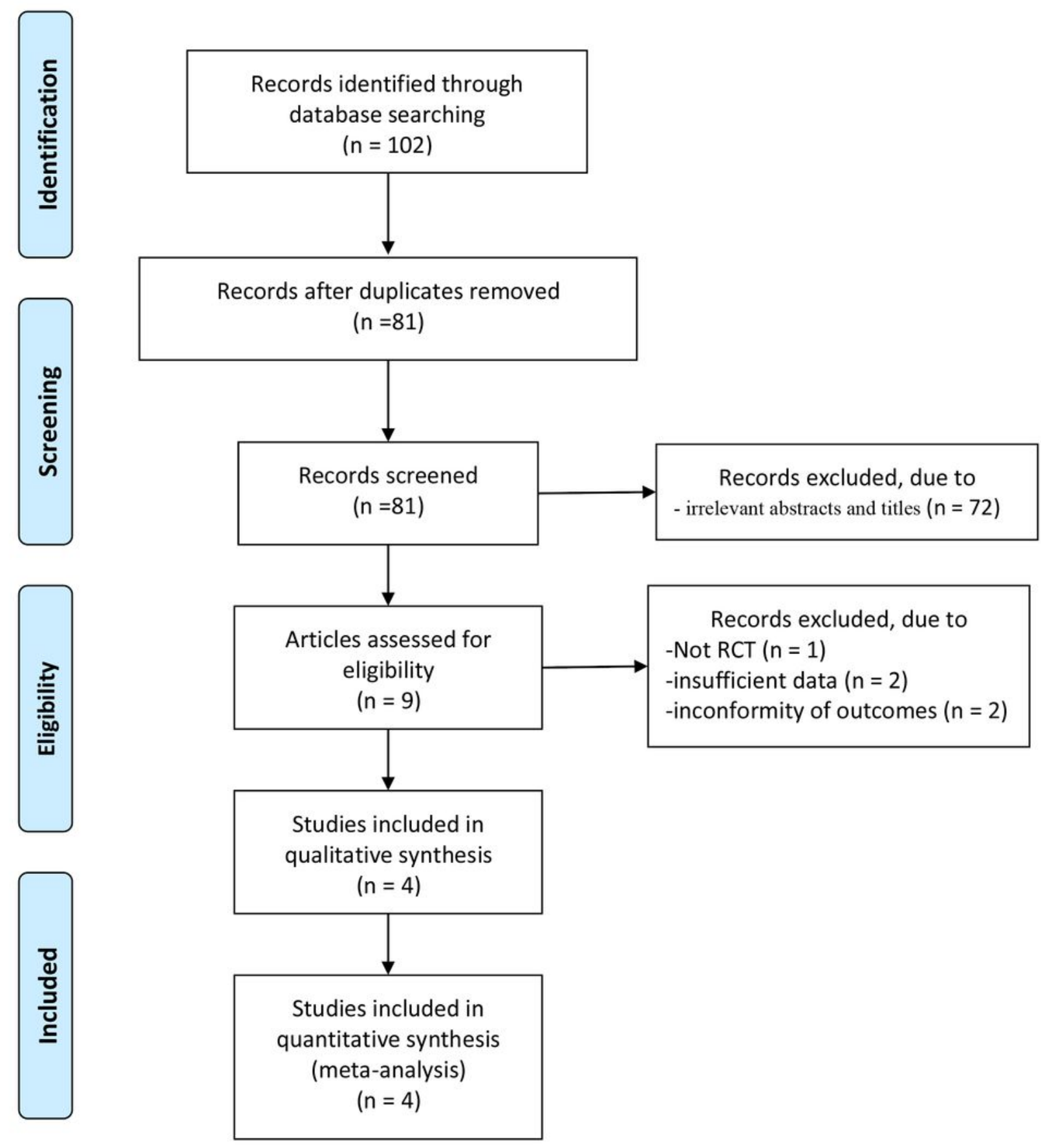

\section{Figure 1}

Flow diagram of study searching and selection process. 
Experimental Control Risk Ratio

Risk Ratio

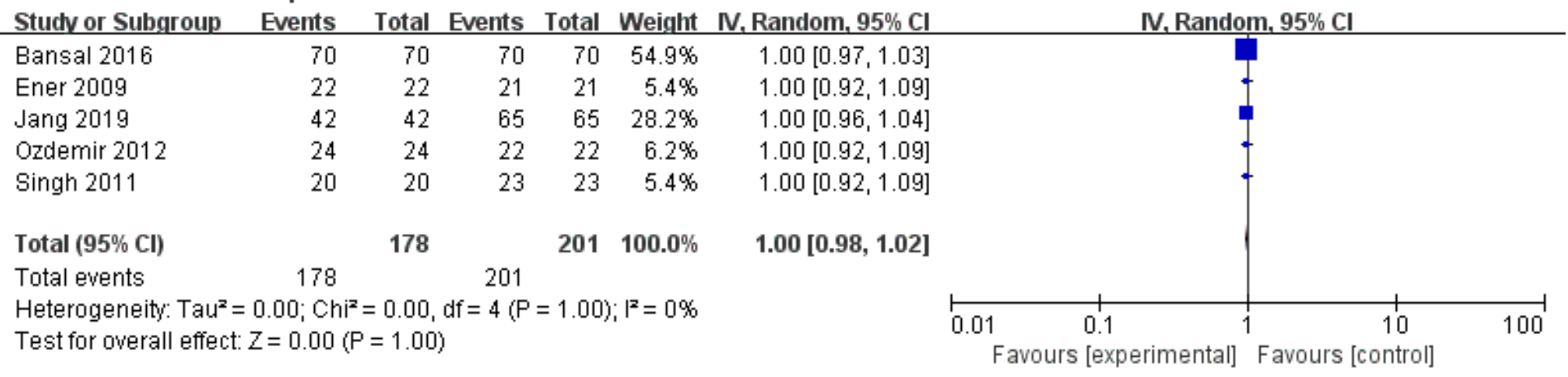

\section{Figure 2}

Forest plot for the meta-analysis of stone clearance.

\begin{tabular}{|c|c|c|c|c|c|c|c|c|c|c|c|c|c|}
\hline \multirow[b]{2}{*}{ Stucty or Subgroup } & \multicolumn{3}{|c|}{ Experimental } & \multicolumn{2}{|c|}{ Control } & & & Mean Difference & \multirow{2}{*}{\multicolumn{5}{|c|}{$\begin{array}{c}\text { Mean Difference } \\
\text { IV, Random, 95\% Cl }\end{array}$}} \\
\hline & Mean & SD & Total & Mean & SD & Total & Weight & IV, Random, $95 \% \mathrm{Cl}$ & & & & & \\
\hline Bansal 2016 & 33.6 & 7 & 70 & 51.2 & 23.2 & 70 & $21.9 \%$ & $-17.60[-23.28,-11.92]$ & & $\rightarrow-$ & & & \\
\hline Ener 2009 & 48.2 & 13.2 & 22 & 68.1 & 22.7 & 21 & $17.9 \%$ & $-19.90[-31.07,-8.73]$ & & - & & & \\
\hline Jang 2019 & 28.8 & 11.9 & 42 & 71.3 & 46.6 & 65 & $17.4 \%$ & $-42.50[-54.39,-30.61]$ & & $\longrightarrow-$ & & & \\
\hline Ozdemir 2012 & 42 & 7.3 & 24 & 59.14 & 10.62 & 22 & $22.1 \%$ & $-17.14[-22.45,-11.83]$ & & -- & & & \\
\hline Singh 2011 & 32.1 & 8.5 & 20 & 69.2 & 16.3 & 23 & $20.6 \%$ & $-37.10[-44.73,-29.47]$ & & $\rightarrow-$ & & & \\
\hline Total $(95 \% \mathrm{Cl})$ & & & 178 & & & 201 & $100.0 \%$ & $-26.26[-35.84,-16.68]$ & & & & & \\
\hline $\begin{array}{l}\text { Heterogeneity: Tau } \\
\text { Test for overall effec }\end{array}$ & $\begin{array}{l}100.64 \\
Z=5.37\end{array}$ & $\begin{array}{l}\mathrm{Chi}^{2}= \\
(\mathrm{P}<0\end{array}$ & $\begin{array}{l}31.88 \\
.00001\end{array}$ & $d f=4($ & $P<0.00$ & $01) ;$ & $=87 \%$ & & -100 & $\begin{array}{l}-50 \\
\text { [experimental }\end{array}$ & ] Favours [c & $\begin{array}{c}50 \\
\text { ontrol] }\end{array}$ & 100 \\
\hline
\end{tabular}

\section{Figure 3}

Forest plot for the meta-analysis of operation time

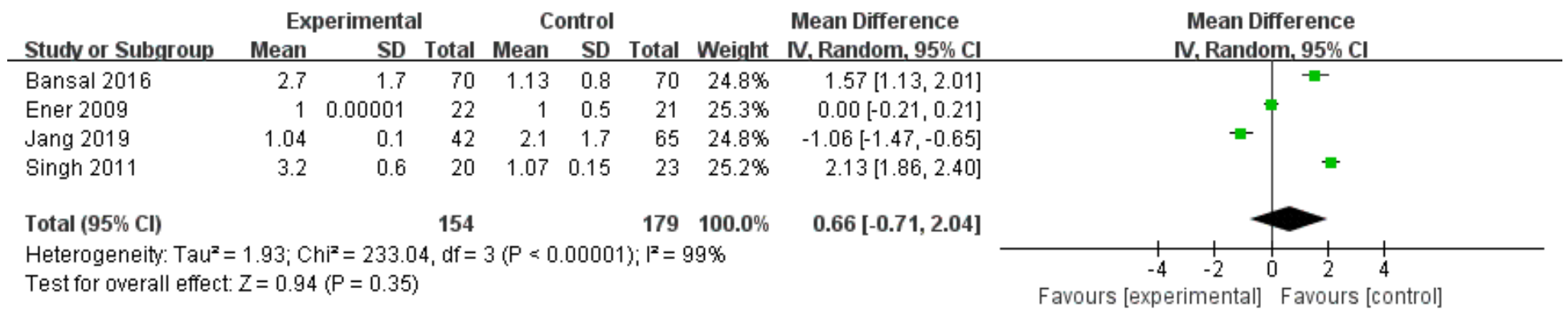

\section{Figure 4}

Forest plot for the meta-analysis of mean urethral entries.

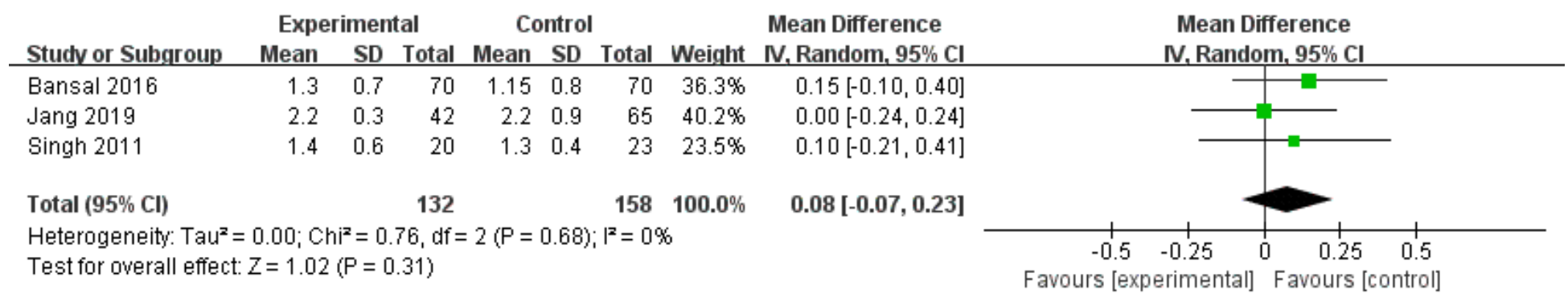


Figure 5

Forest plot for the meta-analysis of hospitalization.

Experimental Control Risk Ratio

Risk Ratio

Studv or Subgroup

Events Total Events Total Weight IV, Random, $95 \% \mathrm{Cl}$

Bansal 2016

Ener 2009

Jang 2019

Ozdemir 2012

Total $(\mathbf{9 5} \% \mathrm{Cl})$

$\begin{array}{lllll}9 & 70 & 5 & 70 & 80.2 \%\end{array}$

$1.80[0.64,5.10]$

Not estimable

$\begin{array}{llllll}0 & 22 & 0 & 21 & \text { Not estimable }\end{array}$

Total events

1

$24 \quad 2 \quad 22 \quad 19.8 \%$

$0.46[0.04,4.71]$

158

$178 \quad 100.0 \%$

$1.37[0.47,4.00]$

Heterogeneity: Tau $^{2}=0.09 ; \mathrm{Chi}^{2}=1.10, \mathrm{df}=1(\mathrm{P}=0.29) ; \mathrm{I}^{2}=9 \%$

Test for overall effect: $Z=0.58$ ( $P=0.56$ )

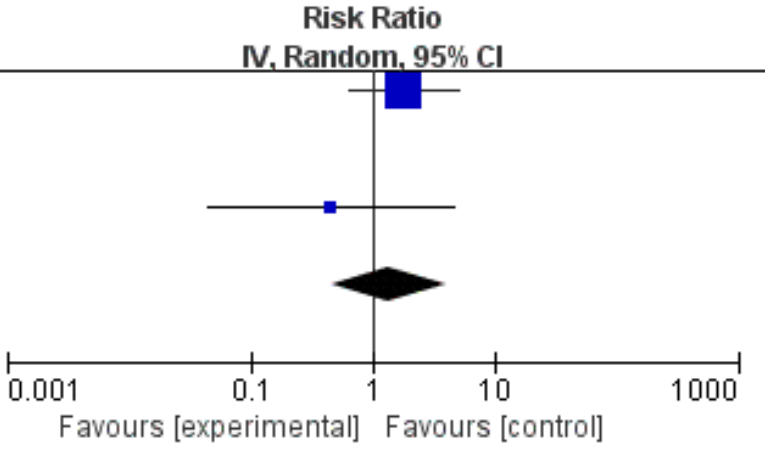

Figure 6

Forest plot for the meta-analysis of total complication. 